\title{
Research Paper: The Effects of Hesperidin on BDNF/TrkB Signaling Pathway and Oxidative Stress Parametersin the Cerebral Cortex of the Utero-placental Insufficiency Fetal Rat Model
}

\author{
Hamed Abdollahi $^{1}$ (D), Mohammad Amin Edalatmanesh ${ }^{1 *}$ (D), Ebrahim Hosseini ${ }^{1}$ (D), Mohsen Foroozanfar ${ }^{2} \mathbb{C}$ \\ 1. Department of Biology, School of Sciences, Shiraz Branch, Islamic Azad University, Shiraz, Iran. \\ 2. Department of Biology, School of Sciences, Marvdasht Branch, Islamic Azad University, Marvdasht, Iran.
}

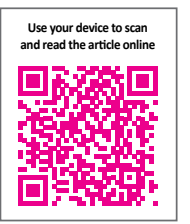

Citation: Abdollahi, H., Edalatmanesh, M. A., Hosseini, E., \& Foroozanfar, M. (2021). The Effects of Hesperidin on BDNF/ TrkB Signaling Pathway and Oxidative Stress Parameters in the Cerebral Cortex of the Utero-placental Insufficiency Fetal Rat Model. Basic and Clinical Neuroscience, 12(4), 511-522. http://dx.doi.org/10.32598/bcn.2021.2187.1

http://dx.doi.org/10.32598/bcn.2021.2187.1

\section{Article info:}

Received: 09 Nov 2019

First Revision: 18 May 2020

Accepted: 13 Jun 2021

Available Online: 01 Jul 2021

Keywords:

Hesperidine, Brain-derived neurotrophic factor, Oxidative stress, Intrauterine growth retardation

\begin{abstract}
AB S T RA C T
Introduction: Uteroplacental Insufficiency (UPI) produces critical neurodevelopmental problems affecting the Intrauterine Growth Restricted (IUGR) in offspring. This study aimed to investigate the possible neuroprotective roles of Hesperidin (Hes) on the fetal cerebral cortex of the UPI rat model.

Methods: In this experimental study, 40 pregnant Wistar rats (age: $\sim 40$ days, Mean \pm SD weight: $180 \pm 10 \mathrm{~g}$ ) were randomly divided into 5 groups $(\mathrm{n}=8 /$ group). The study groups included control (normal saline, orally), UPI+NS (uterine vessel ligation+normal saline, orally), UPI+HES25, UPI+HES50, and UPI+HES100 (uterine vessel ligation+25, 50 and 100 $\mathrm{mg} / \mathrm{kg}$ Hes, orally). After being anesthetized by ketamine and xylazine, UPI was induced by permanent bilateral closure of the uterine vessels on Gestation Day (GD) 18. From GD15, the Hes/NS-treated groups received Hes/normal saline until GD21. On GD21, the uterus, placenta, and fetus were dissected out and weighed. The oxidative stress parameters, including Catalase (CAT) activity, Malondialdehyde (MDA), and Total Antioxidant Capacity (TAC) were measured in the fetal cerebral cortex. The expression of Brain-Derived Neurotrophic Factor (BDNF) and Tropomyosin Receptor Kinase B (TrkB) was assessed by RT qPCR methods. The obtained data were analyzed by Analysis of Variance (ANOVA) and Tukey's post hoc test.

Results: The present study findings identified a significant difference in the uterine and fetus weight in Hes-treated mothers $(\mathrm{P}<0.05)$. In the fetus, Hes reduced MDA, and increased CAT activity and TAC $(\mathrm{P}<0.001$ in the UPI+Hes 100 group, compared to the UPI+NS group). UPI reduced $\mathrm{BDNF}$ and TrkB mRNA expression, compared to the control group $(\mathrm{P}<0.05)$. Also, Significant increases in BDNF and TrkB mRNA expression were observed after administrating Hes in the fetal cerebral cortex of the UPI rat model, in a dose-dependent manner $(\mathrm{P}<0.05)$.

Conclusion: Hes, as a neuroprotective and antioxidant agent, accelerates BDNF-TrkB signaling pathway and suppresses oxidative stress parameters in the cerebral cortex of the UPI rat model.
\end{abstract}

\section{* Corresponding Author:}




\section{Highlights}

- Hesperidin accelerates the BDNF-TrkB signaling pathway.

- Improved BDNF-TrkB signaling pathway can ameliorate neurodegenerative diseases.

- Hesperidin suppresses oxidative stress parameters in the fetal cerebral cortex of the UPI rat model.

- Hesperidin is effective in reducing cerebral cortex damage with its antioxidant role.

- Hesperidin can be effective in reducing UPI-induced cerebral cortex damage.

\section{Plain Language Summary}

The abnormal fetal growth etiology and its mechanism, i.e., Intrauterine Growth Restriction (IUGR), remain unclear. IUGR produces significant neurodevelopmental deficits affecting the IUGR offspring's cerebral cortex. Furthermore, compounds, including Hesperidin (Hes) (of different antimicrobial \& antioxidant properties) might present neuroprotective effects. In this study, initially, the UPI model was created by bilateral uterine vessel ligation, then the study rats were given oral Hes. Hes caused uterine and embryo weight gain in the UPI model. Analyzing antioxidant parameters suggested that the Catalase (CAT) activity and Total Antioxidant Capacity (TAC) increased, and Malondialdehyde (MDA) decreased after Hes administration in the fetal cerebral cortex. BDNF plays a crucial role in brain development, including neural migration, neurogenesis, and synapse formation in the antenatal and postnatal. Hesperidin also increased BDNF and its receptor, TrkB, in the UPI model. In general, the collected results indicated that Hes could effectively reduce the IUGR-induced cerebral cortex damage. Additionally, Hes may provide the beneficial therapeutic potential to inhibit and treat the neurodegenerative disorders associated with intrauterine growth restriction.

\section{Introduction}

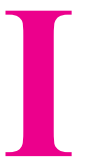

ntrauterine Growth Restriction (IUGR) is generally determined as the remarkable lessening in fetal growth rate, resulting in low birth weight. It affects $10 \%$ of all pregnancies. Besides, IUGR is a major contributor to perinatal mortality and morbidity (20\%$50 \%$ of perinatal deaths). IUGR is also related to aggravated long- and short-term outcomes as the prevalence of intrapartum distress, suboptimal neurodevelopment, neonatal complications, and increased odds of cardiovascular disease (Gumus et al., 2018). The chronic reduction of placental blood supply induced by continued exposure to under-nutrition and hypoxemia causes subsequent consequences on brain development (Illa et al., 2013). The association between neurodevelopmental dysfunctions and IUGR was widely described. During postpartum, the term IUGR presents worse cognitive performance and neurobehavioral, compared to control term infants. Furthermore, reports suggested that infants born with IUGR have learning difficulties in school concerning a characteristic pattern involving attention, short-term memory, and anxiety (Geva, Eshel, Leitner, Fattal-Valevski, \& Harel, 2006). Uteroplacental Insuffi- ciency (UPI) is a model of Intrauterine Growth Restriction (IUGR) caused by characteristics, such as maternal smoking, preeclampsia, and the abnormalities of uteroplacental development (Pinney, 2016). UPI produces significant neurodevelopmental deficits affecting the cerebral cortex of IUGR offspring. The structure, frequency, and shape of the various cerebral cortex cells vary in the offspring of IUGR; however, the precise mechanism of these events remains unclear (Fung et al., 2012).

Hesperidin (Hes), a bioflavonoid, is the major flavanone present in citrus fruits, including orange. It is composed of Hes being conjugated to rutinose. In the intestine following Hes administration, this compound is absorbed through the paracellular pathway by conversion to Hes. The Hes that is part of the flavonoids acts in plants as antimicrobials, antioxidants, visual attractors, photoreceptors, feeding repellants, and is used for light screening (Estruel-Amades et al., 2019). Hes has different biological activities, e.g., this bioflavonoid is a potentially protective marker for neurodegenerative diseases; i.e., achieved by reducing neuroinflammation in experimental stroke (Kawser Hossain et al., 2016). The majority of the flavonoids found in citrus fruits are glycosides and only a small quantity of Hes is present. Numerous pharmaco- 
logical effects of Hes have been described. Hes prevents hepatic steatosis, lipid-droplet accumulation, and other disorders, including hypercholesterolemia, hypertension, osteoporosis, and cerebral thrombosis, among others (Estruel-Amades et al., 2019). The role of Hes was reported in neuroprotective efficacy. The Hes-related neuroprotective efficacy is attributed to its inhibition ability of Reactive Oxygen Species (ROS) generation, including scavenging peroxynitrite radicals, hydroxyl radical, and inhibiting $\mathrm{Fe}^{2+}$-induced linoleate peroxidation, and cerebral membranes auto-oxidation. It protects the neurons against various assaults associated with multiple neurodegenerative diseases (Raza et al., 2011).

Neurotrophins are a group of associated proteins, i.e., first recognized as survival markers for sympathetic and sensory neurons. They were reported to control various dimensions of development, survival, and function of neurons in both central and peripheral nervous systems. These proteins also control axon growth, cell fate decisions, dendrite pruning, and growth, as well as protein expressions, such as transmitter biosynthetic enzymes, ion channels, and neuropeptide transmitters that play a vital role in healthy neuronal function. Neurotrophins also provide supplemental, subtler functions outside the nervous system (Reichardt, 2006). The Brain-Derived Neurotrophic Factor (BDNF) is a neurotrophin family member, one of the neurotrophic factors, and a secretion protein. It increases the differentiation speed of neuronal populations of central and peripheral nervous systems (Verma, Sharma, Sarma, Chetia, \& Saikia, 2013). It plays a critical role in regulating neuronal survival, as well as maintenance, development, the regulation of the formation, storage, and cognition of memories. The extents of BDNF and its receptors are decreased in the cerebral cortex of normal aging animals and Alzheimer's disease; this might contribute to cellular and synaptic loss and memory deficits (Miranda et al., 2018). The neurotrophic effects of BDNF are mainly facilitated through binding to its receptor, Tropomyosin Receptor Kinase B (TrkB). $\mathrm{BDNF}$ and its receptor, TrkB, are mostly expressed in the mammalian brain. BDNF/TrkB-stimulated signaling is crucial for neuronal morphogenesis, plasticity, and survival (Dekeyster et al., 2015). Hes could effectively protect neurons from injuries induced by nitrosative or oxidative stress. Moreover, it enhances cognitive functions through different mechanisms, including elevating BDNF (Hajialyani et al., 2019).

It is important to characterize the potential for restoring normal neurodevelopment after chronic reductions in nutrient supply, which occur in the uterus, and to understand the mechanisms that might lead to improved brain function. Therefore, this study aimed to determine the Hes inhibitory effects on brain damage in the Uteroplacental Insufficiency (UPI) model. Moreover, this study examined the inhibitory effects of Hes on BDNF and its receptor, TrkB in the cerebral cortex (neocortex) of the UPI rat model.

\section{Methods}

All protocols of the current study were approved by the Committee for Animal Research of Islamic Azad University, Shiraz branch. Animal experiments were performed as per the Guide for the Care and Use of Laboratory Animals. All study animals were housed at $\sim 50 \%$ relative humidity and ambient temperature of $22 \pm 3{ }^{\circ} \mathrm{C}$, with free access to water and food, and a 12:12 hour light/dark cycle. In this laboratory study, 40 pregnant Wistar rats (age: $\sim 40 \mathrm{~d}$, Mean \pm SD weight: $180 \pm 10 \mathrm{~g}$ at gestation day 1) were used. The examined rats were randomly divided into 5 groups ( $\mathrm{n}=8$ /group), including control, UPI+NS, UPI+HES25, UPI+HES50, and UPI+HES100. In the control group, the laparotomy surgery was performed except that uterine vessels were not ligated. Then, mothers were treated with 0.5 ccs normal saline as HES solvent. In the UPI groups, the uteroplacental insufficiency surgery was performed on GD18 (term $\sim 22$ days) as described previously by Wlodek et al. (2005). Briefly, on GD 18 and under deep anesthesia by intraperitoneal injection of ketamine $(50 \mathrm{mg} / \mathrm{kg})$ and xylazine $(10 \mathrm{mg} /$ $\mathrm{kg}$ ), laparotomy surgury was conducted. Then, bilateral uterine vessels (vein \& artery) were ligated which restricted nutrient and blood supply delivery to the fetuses.

The explored animals were anesthetized on the $21^{\text {st }}$ day of gestation. Then, the uterus, placenta, and fetus were dissected out and weighed using a digital scale (Kern, Germany). The live fetus was put fetus on an ice-cold surface, then the head was removed. The anterior brain was recognized under stereo microscopes (Olympus, Tokyo, Japan). The anterior brain was rapidly isolated and washed by ice-cold isotonic saline. Next, the cerebral cortex was separated until the next steps, i.e., storage at $-80^{\circ} \mathrm{C}$. The frozen tissues were used to extract RNA or prepare tissue homogenates to evaluate oxidative stress parameters. Accordingly, cerebral cortex tissues were homogenized in a lysis buffer (Triton X-100, NaCl, Tris, EDTA, SDS, EGTA, HEPES, \& PMSF) (all chemicals were purchased from Merck, Germany) using a homogenizer (T10Bhomogenizer; Germany), and were centrifuged for $45 \mathrm{~min}\left(13000 \mathrm{rpm}\right.$ at $\left.4{ }^{\circ} \mathrm{C}\right)$. Then, the supernatant was used to measure the antioxidant activity. 
Table 1. Primers used in the present study

\begin{tabular}{ccc}
\hline Genes & Primer Sequences & Sizes (bp) \\
\hline \multirow{2}{*}{$\beta 2 \mathrm{~m}$} & Forward: 5'-CGTGCTTGCCATTCAGAAA -3' & 244 \\
& Reverse: 5'-ATATACATCGGTCTCGGTGG -3' & 120 \\
\hline Bdnf & Forward: 5'- GAACGGGAGGGGTAGATTTC -3' & 169 \\
\hline TRKB & Reverse: 5'- CAACCAGAATGGAGAGTGAAGA -3' \\
\hline
\end{tabular}

Catalase (CAT) is also known as hydrogen peroxide oxidoreductase. Briefly, the supernatant was diluted with $0.05 \mathrm{M}, \mathrm{pH} 7.0$ phosphate buffer, i.e., used and incubated at room temperature for $10 \mathrm{~min}$. The supernatant was administered to evaluate the CAT activity by a commercial kit (ZB-CAT ZellBio A 96, Germany) according to the manufacturer's instructions and was read by a microplate/ELISA reader (Micro Plate Reader, Germany). One unit corresponds to $1 \mathrm{nmol}$ CAT per gram tissue $(\mathrm{U} / \mathrm{mL})$.

The MDA level was calculated by the reverse phase HPLC-MS method using the single ion monitoring technique. The chemicals used for spectrophotometric and HPLC-MS determination of MDA-TBA included Thiobarbituric Acid (TBA) and Butylated Hydroxytoluene (BHT) (Sigma Aldrich, USA). Ammonium acetate, potassium dihydrogen phosphate, and Trichloroacetic Acid (TCA) were obtained from Merck (Merck, Germany). From that stock, an aliquot of $500 \mu \mathrm{L}$ was transferred into a $5 \mathrm{mLNunc}$ Cryotube, and $1 \mathrm{~mL}$ TBA $(0.375 \%$ in $0.25 \mathrm{MHCl}$ ) was added. Then, the mixture was heated at $100^{\circ} \mathrm{C}$ for 15 minutes. After cooling, the solution was colorimetrically analyzed by spectrophotometry using a $1 \mathrm{~cm}$ absorption cell (Hitachi, Japan) at 530-540 nm (Lunec, 1990). A standard curve was used to calculate the MDA concentration. MDA levels were expressed as $\mu \mathrm{mol} / \mathrm{L}$ concentrations using the curves of calibrating. They were expressed as a decreased percentage in MDA level compared to the negative control.

The total antioxidant capacity of cerebral cortex homogenates was estimated by the TAC kit (ZB-TAC ZellBio A 96, Germany) according to the manufacturer's instructions. A certain amount of PBS (pH 7.4) was added. Next, the sample was thoroughly homogenized by a homogenizer. The absorbance of the supernatant was then determined at 490nm using an ELISA plate reader. The obtained results were evaluated from the standard curve and expressed as millimoles per gram protein.
Total RNA was isolated from the cerebral cortex using the RNA extraction kit (Cinnagen Inc., Iran). The optical density 260/280 was measured using a Nanodrop ${ }^{\mathrm{TM}}$ spectrophotometer (Nanodrop; Thermo Fisher Scientific, Wilmington, DE, USA) for the concentration and purity of RNA. Complementary DNA (cDNA) was synthesized from $1 \mu \mathrm{g}$ of RNA using the RevertAid ${ }^{\mathrm{TM}}$ firststrand cDNA synthesis kit (Thermo Fisher Scientific, Inc., Waltham, MA, USA). Real-Time PCR was conducted in the applied Biosystems StepOne ${ }^{\mathrm{TM}}$ instrument (ABI, Step One, USA) similar to the protocol of RealQ Plus 2x Master Mix Green (Amplicon A/S, Odense, Denmark). As per Table 1, real-time PCR for the expression analysis of the primer pairs for BDNF, TrkB, and beta-2 microglobulin (b2m) was designed by the Primer3 software. The $(\mathrm{b} 2 \mathrm{~m})$ reference gene was also used as the real-time PCR reactions internal control. The conditions of real-time PCR were set for 10 minutes at $94^{\circ} \mathrm{C}$, followed by 40 cycles of $15 \mathrm{sec}$ at $94^{\circ} \mathrm{C}$; one minute at $60^{\circ} \mathrm{C}$ in annealing and extension steps that continued with the melting curve step. The melting curve analysis and gel electrophoresis were performed to confirm targets specific amplification after each run of real-time PCR. The different samples amplification signals were normalized to (b2m) Cycle Threshold $(\mathrm{Ct})$. Subsequently, the delta-delta CT (2- $\Delta \Delta \mathrm{CT})$ method was applied to compare the test mRNA levels to the control, which was demonstrated as a fold change in the data analysis.

Between-Group comparisons were performed using one-way Analysis of Variance (ANOVA). The normality of quantitative variables was analyzed by the Kolmogorov-Smirnov test and the normally distributed variables were compared to variance analyses and Tukey's post hoc tests. Differences were considered significant at $\mathrm{P}<0.05$. All statistical analyses were conducted in SPSS. The collected results were expressed as Mean \pm Standard Deviation (SD) unless otherwise noted. 


\section{Results}

Figure 1 shows the Mean \pm SD values of uterine, fetus, and placenta weights. The Kolmogorov-Smirnov test results indicated that the distribution of the results is normal. The results of one-way ANOVA identified a significant difference in the levels of uterine weight $\left(\mathrm{F}_{4,30}=\right.$ 25.74, $\mathrm{P}<0.001)$ and fetus weight $\left(\mathrm{F}_{4,30}=7.80, \mathrm{P}<0.001\right)$ between the research groups. However, there was no significant difference in the weight of the placenta $\left(\mathrm{F}_{4,30}=\right.$ $2.56, \mathrm{P}=0.051)$.

The results of Tukey's post hoc test indicated that UPI caused an increase in the weight of the uterine in the
UPI+NS group, compared to the controls $(\mathrm{M}=-2.09$, $\mathrm{P}<0.001$ ). Additionally, Tukey>s post hoc test data revealed that the Hes-treated groups presnted no significant difference, compared to the UPI+NS group (UPI+Hes25 vs. UPI+NS: $\mathrm{M}=-1.35, \mathrm{P}=0.068$; $\mathrm{UPI}+\mathrm{Hes} 50$ vs. UPI+NS: $\mathrm{M}=-0.46, \quad \mathrm{P}=0.65, \& \mathrm{UPI}+\mathrm{Hes} 100$ vs. UPI+NS: $\mathrm{M}=-1.21, \mathrm{P}=0.58)$. There was no significant difference in the levels of uterine weight between the Hes-treated groups.

The results of Tukey's post hoc test suggested that UPIsignificantly impacted fetal weight loss in rats (UPI+Ns vs. the controls: $\mathrm{M}=2.50, \mathrm{P}<0.001)$. However, the UPI+Hes25 group $(\mathrm{M}=-0.89, \mathrm{P}=0.10)$ and the
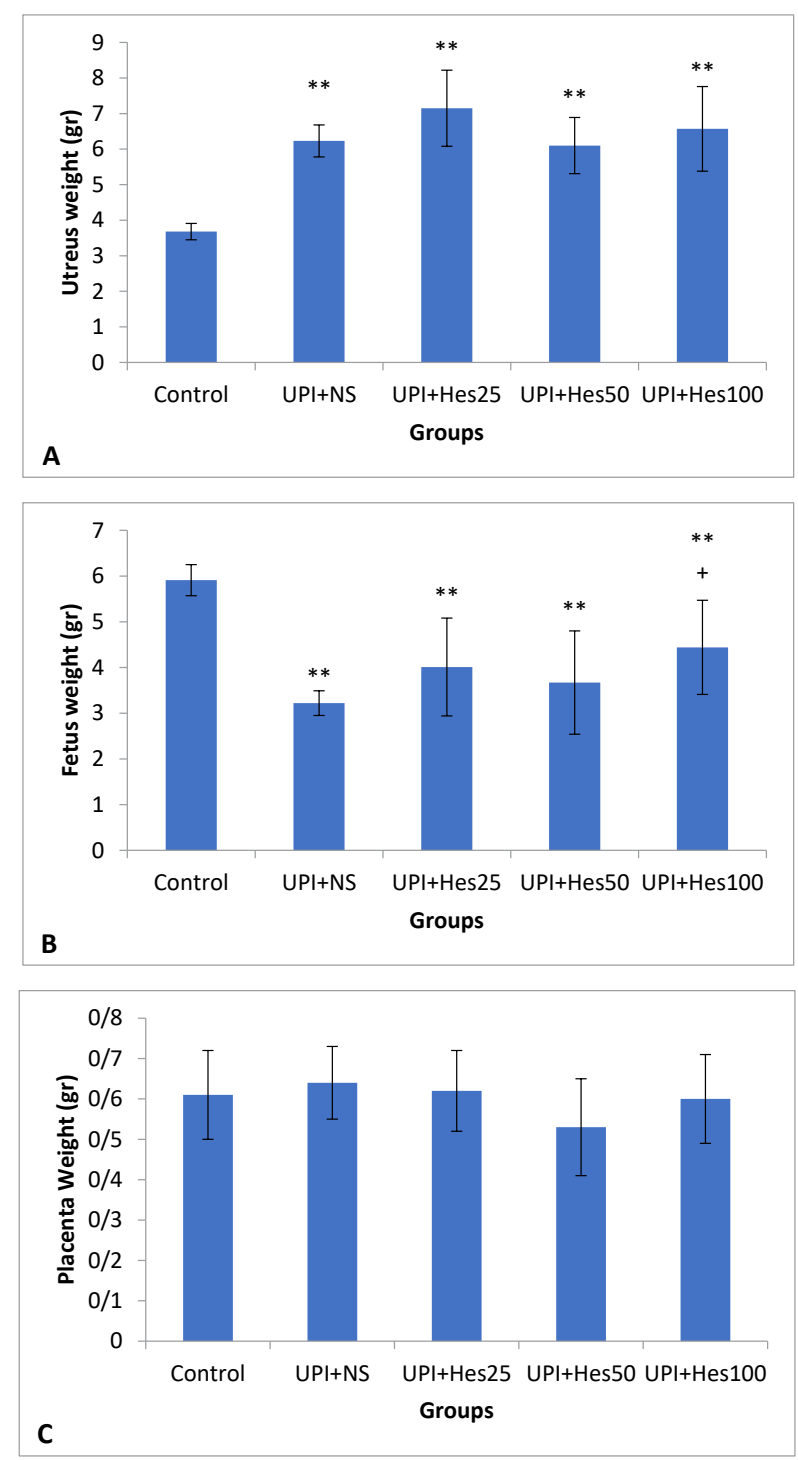

Figure 1. The effect of Hesperidin $(\mathrm{H})$

A: Weight of the uterus; B: The fets; and C: The placenta of the rat (gram). Data are presented as Mean \pm SD of ten animals.

$\mathrm{P}<0.05$ compared to the control group presented by $\left({ }^{*}\right)$, the $\mathrm{P}<0.05$ compared to the UPI+NS group presented by $(+) ;{ }^{+} \mathrm{P}<0.05$ and ${ }^{*} \mathrm{P}<0.001$ 

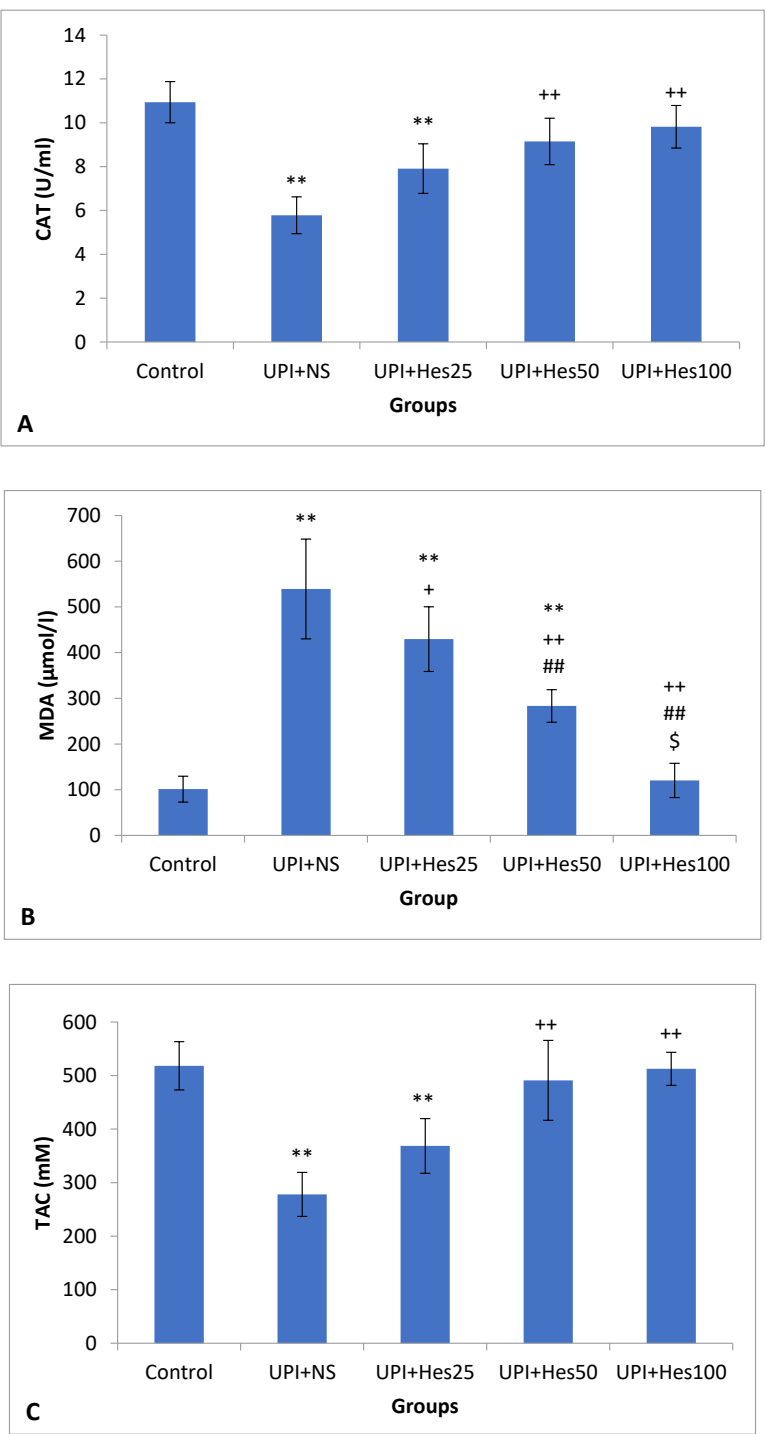

Figure 2. The oxidative stress parameters in the cerebral cortex

The effect of Hes on A: Catalase (CAT, U/ml); B: Malondialdehyde (MDA, $\mu \mathrm{mol} / \mathrm{L})$; and C: Total antioxidant capacity (TAC, $\mathrm{mM}$ ) levels. Data are presented as Mean \pm SD. $\mathrm{P}<0.05$ compared to the control group presented by $\left({ }^{*}\right)$, the $\mathrm{P}<0.05$ compared to the UPI+NS group presented by (+), the $\mathrm{P}<0.05$ compared to the UPI+CA25 group presented by (\#) and the $\mathrm{P}<0.05$ compared

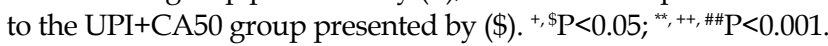

UPI+Hes50 group $(\mathrm{M}=-0.55, \mathrm{P}=0.55)$ presented no significant difference, compared to the UPI+NS group. However, the UPI+Hes100 group provided a significant effect on fetal weight gain, compared to the UPI+NS group $(\mathrm{M}=-1.32, \mathrm{P}=0.04)$. There was no significant difference between the UPI+Hes50 $(\mathrm{M}=-0.33, \mathrm{P}=0.81)$, $\mathrm{UPI}+$ Hes $100(\mathrm{M}=-0.43, \mathrm{P}=0.84)$, and UPI+Hes 25 groups. Furthermore, there was no significant difference between the UPI+Hes50 and the UPI+Hes100 groups $(\mathrm{M}=-0.76, \mathrm{P}=0.37)$. Figure 2 presents the results for the antioxidant capacity of Hes. The collected results revealed the normal distribution of the study data. The oneway ANOVA data determined a significant difference in the CAT activity $\left(\mathrm{F}_{4,30}=14.95, \mathrm{P}<0.001\right)$, the tissue level of MDA $\left(\mathrm{F}_{4,30}=26.12, \mathrm{P}<0.001\right)$, and TAC $\left(\mathrm{F}_{4,30}=\right.$ $32.49, \mathrm{P}<0.001)$. The UPI significantly declined CAT activity in the cerebral cortex of UPI+NS, compared to the control group $(\mathrm{M}=2.25, \mathrm{P}<0.001)$. However, the $\mathrm{UPI}+\mathrm{Hes} 25$ group presented no significant increase in the CAT activity, compared to the UPI+NS group (M= $-1.15, \mathrm{P}=0.29$ ); however, CAT activity was higher in the UPI+Hes50 $(\mathrm{M}=-2.38, \mathrm{P}=0.002)$ and $\mathrm{UPI}+\mathrm{Hes} 100$ $(\mathrm{M}=-4.17, \mathrm{P}<0.001)$ groups, compared to the UPI+NS group. The results of Tukey's post hoc test indicated that UPI significantly increased MDA levels in the cerebral cortex (UPI+NS group vs. the controls: $\mathrm{M}=-548.83$, 

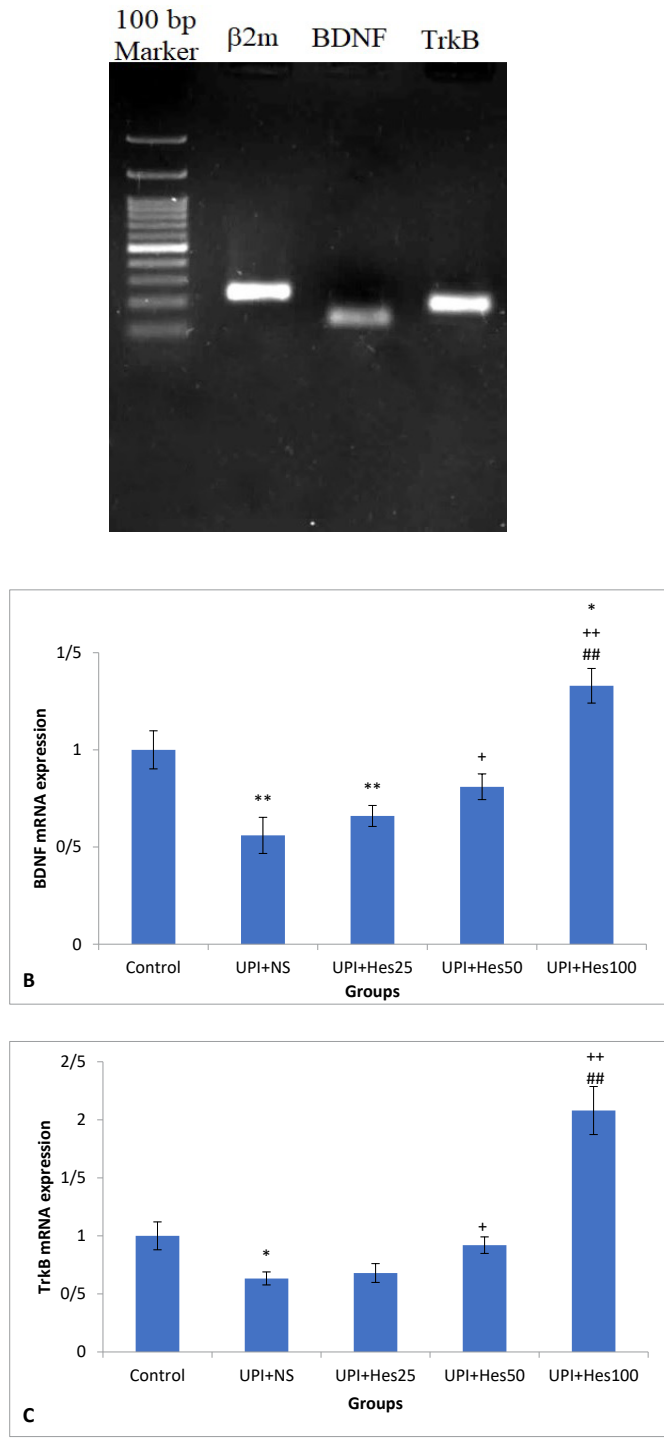

NEUROSCIENCE

Figure 3. Gel electrophoresis and effect of hesperidin (Hes) on BDNF and TrkB genes expression in the cerebral cortex of the UPI rat model

A: Gel electrophoresis for $\beta 2 \mathrm{~m}, \mathrm{BDNF}$ and TrkB genes; B:The BDNF; and C: TrkB genes expression levels were estimated by the quantitative real-time PCR in Hes-treated groups (UPI+Hes25, UPI+Hes50 and UPI+Hes100). Expression data relative to those of the reference gene are presented as Mean \pm SEM. Statistical significance was tested using the one-way ANOVA. P $<0.05$ compared to the control group presented by $\left(^{*}\right)$, the $\mathrm{P}<0.05$ compared to the UPI+NS group presented by $(+)$, the $\mathrm{P}<0.05$ com-

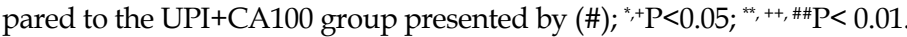

$\mathrm{P}<0.001)$. Furthermore, the tissue level of MDA significantly decreased in the UPI+Hes $25(\mathrm{M}=209.63$, $\mathrm{P}<0.01)$, UPI+Hes50 $(\mathrm{M}=355.90, \mathrm{P}<0.001)$, and the UPI+Hes100 (M= 518.83, $\mathrm{P}<0.001)$ groups, compared to the UPI+NS group. There was a significant decrease of MDA in the UPI+Hes100 group, compared to the $\mathrm{UPI}+\mathrm{Hes} 25(\mathrm{M}=309.19, \mathrm{P}<0.001)$ and $\mathrm{UPI}+\operatorname{Hes} 50(\mathrm{M}=$ $232.15, \mathrm{P}<0.001)$ groups. Moreover, there was a significant difference between the UPI+Hes 25 and UPI+Hes50 groups $(\mathrm{M}=122.52, \mathrm{P}=0.002)$. The result of Tukey's post hoc test for TAC addressed that UPI provided a sig- nificant effect on TAC reduction in the examined rats' cerebral cortex (UPI+NS group vs. the controls: $\mathrm{M}=$ 293.76, $\mathrm{P}<0.001)$. However, there was no significant difference in the UPI+Hes25 group, compared to the UPI+NS group $(\mathrm{M}=-88.15, \mathrm{P}=0.052)$. The TAC level significantly increased in the UPI+Hes50 $(\mathrm{M}=-210.67$, $\mathrm{P}<0.001)$ and $\mathrm{UPI}+\mathrm{Hes} 100 \quad(\mathrm{M}=-232.16, \quad \mathrm{P}<0.001)$ groups, compared to the UPI+NS group.

The analysis of the real-time quantitative PCR aimed to compare the level of BDNF and TrkB genes expres- 
sion, in response to Hes-treated different concentrations (UPI+Hes25, UPI+Hes50, \& UPI+Hes100 groups). The expression analysis data indicated that the BDNF expression in the cerebral cortex of the study rats was significantly down-regulated after UPI (0.56 fold in the UPI+NS group, compared to the controls, $\mathrm{P}=0.01$ ). The achieved results determined that the level of BDNF gene expression increased in a dose-dependent manner respecting Hes. The BDNF gene expression increased in the UPI+Hes50 and UPI+Hes100 groups ( 0.81 fold \& 1.33 fold changes, respectively), compared to the UPI+NS group $(\mathrm{P}=0.04 \& \mathrm{P}<0.01$, respectively).

However, in the UPI rat model, TrkB gene expression was reduced in the UPI+NS group ( 0.63 folds), compared to the control group, $(\mathrm{P}=0.016)$. As per the result of real-time quantitative PCR results, the highest levels of TrkB gene expression were found in the UPI+Hes 100 groups, followed by the UPI+Hes50 groups (2.08 fold \& 0.92 fold changes, respectively), compared to the UPI+NS group $(\mathrm{P}<0.001 \& \mathrm{P}=0.010$, respectively). There was no significant difference in the expression of TrkB between the UPI+Hes 25 groups ( 0.68 folds) and the UPI+NS group. As per Figure 3, the collected data suggested that Hes, in different concentrations served, as an effective inducer of cell-protective through the activation of neuroprotective genes.

\section{Discussion}

This study demonstrated that UPI and the consumption of Hes, as a potent antioxidant are associated with weight changes in the fetus, uterus, and placenta, the alteration of oxidative stress parameters in the fetal cerebral cortex, and the expression increase of BDNF and TrkB gene expression in the Hes-treated groups. The main result of this study was that, in acute UPI-operated rats, hippocampal antioxidant capacity was decreased in the rat IUGR offspring. The present study observed the beneficial effects of Hes on the weight of the uterus and the fetus and the expression of neuroprotective genes (BDNF \& TrkB) in the neocortex of the UPI rat model. Previous studies suggested that uteroplacental insufficiency was associated with neurological difficulties in the CNS (Deodati et al., 2018; Fung et al., 2012). In the IUGR model, rat offspring demonstrated a decrease in the number of hippocampal neurons at birth (Fung et al., 2012; Tashima, Nakata, Anno, Sugino, \& Kato, 2001). The present study data indicated that Hes played a role in fetal and uterine weight. Previous studies provided the first evidence for stimulatory effects of flavonoids on the development and growth of Porcine Trophectoderm ( $p$ Tr) cells in vitro. Besides, these results suggested that flavonoids may affect the development of the porcine fetus in vivo and increase reproductive performance concerning successful outcomes of pregnancy, embryonic, and fetal survival (Lim \& Song, 2016).

General food restriction is a trigger for IUGR. Evidence for environmental factors and maternal lifestyle that could impact neonatal BDNF is limited. For example, mothers who smoke during pregnancy have higher cord serum levels of BDNF, but only among girls (Spulber et al., 2010). Maternal mental disorders are related to lower BDNF levels in umbilical cord blood. Furthermore, the impacts of maternal weight and adiposity on children's neurodevelopment were indicated (Ghassabian et al., 2017). BDNF was involved in some neuropsychiatric disorders, such as autism spectrum disorder, depression, schizophrenia, and epilepsy; thus, the association between neonatal BDNF levels and children's neurodevelopmental outcomes was interesting (Molendijk et al., 2014).

Investigations revealed that, by binding the BDNF to the TrkB receptor and dimerizing this receptor, as well as activating NMDA receptors, 3 types of intracellular cascade signals are generally triggered, as follows: the PLC- $\gamma$ pathway activating the Protein Kinase C (PKC), the PI3K pathway activating the serine/trounine kinase AKT, and MAPK or ERK pathway activating several downstream factors. Each of these pathways produces a unique function of BDNF on the cells (Meng et al., 2019). Neonatal BDNF was used as a marker of plasticity to assess the influence of prenatal exposures on fetal neurodevelopment in human and animal research. Animal studies revealed that BDNF overexpression in the rat's cerebral cortex can play a crucial role in a reactive BDNF response and neuroprotective mechanism of the CNS (Boschen, Criss, Palamarchouk, Roth, \& Klintsova, 2015). The present study indicated that Hes could increase the expression of the BDNF gene in the neocortex. The cerebral cortex is among the brain areas with high BDNF gene expression. Moreover, factors affecting BDNF expression in the cerebral cortex may influence learning, memory, and cognitive information necessary to control food intake in pregnancy (Ghassabian et al., 2017). BDNF plays an important role in brain development, including neural migration, neurogenesis, and synapse formation in antenatal and postnatal stages. Various animal studies suggested that BDNF and other neurotrophins may involve the pathogenesis of neurodevelopmental delays (Janke, Cominski, Kuzhikandathil, Servatius, \& Pang, 2015).

The active components of medicinal plants have long been used to improve mental function. These compounds, in addition to their unique properties, such as anti-neuroinflammatory and antioxidant activities, are 
promising agents to treat neurodegenerative diseases (Furlan, Konc, \& Bren, 2018). Several components were considered potential neuroprotective agents in the laboratory. Hes is among the main flavones in citrus fruits. Citrus flavonoids have anti-inflammatory and antioxidant bioactivities. In vivo and in vitro studies revealed that citrus flavonoids exert neuroprotection and can even promote cognition. The benefits of these compounds, especially Hes, on brain health include bioavailability and high safety, multiple neuroprotective mechanisms, and the transversion of the blood-brain barrier (Hwang, Shih, \& Yen, 2012).

The physiological concentrations of Hes can initiate several intracellular signaling pathways and induce proteins that prevent anti-oxidative stress, promote cognition, or are resistant to $A \beta$-associated neurotoxicity (Tamilselvam et al., 2013). Owing to the neuroprotective effects of hesperidin, different results dedicated their effort to explore the molecular target and neuropharmacological mechanisms of hesperidin, among the citrus flavonoids. Hes can exert antioxidant effects by increasing the expression of CAT, Glutathione (GSH) peroxide, Mn-SOD, and glutathione reductase (Tamilselvam et al., 2013). Researchers concluded that except for radical scavenging activity, increasing the defenses of the antioxidant cellular via an ERK/Nrf2 signaling pathway was an additional mechanism essential for citrus flavonoids' anti-oxidative effects, including Hes (Parhiz, Roohbakhsh, Soltani, Rezaee, \& Iranshahi, 2015).

A high dose of Hes protected $A \beta$-associated neurotoxicity, cortical neurons were against oxidative stress, glutamate-induced excitotoxicity, and decreased the oxidation of lipids and proteins (Tamilselvam et al., 2013). The levels of antioxidant enzymes, including SODs, CAT, GSH peroxide, and reductase, were augmented. Additionally, a study reported that consuming Hes restricts the rat brain damage via declining inflammation and free radicals following stroke (Raza et al., 2011). Furthermore, Hes inhibits NMDA toxicity. Based on the obtained findings, the NMDA receptor pathway may be the target site of Hes to exert neuroprotective action against excitotoxicity. The inhibition of excitotoxicity can be due to the high antioxidant capacity of Hes (Cho, 2006). The present study revealed that Hes, in high concentrations, led to a significant increase in TrkB gene expression. Other studies signified that Hes can trigger Estrogen Receptors (ERs); in turn, estrogen via ER and Trks mediated signaling display neuroprotective effects. Furthermore, citrus flavonoids, including Hes activated MAPK/ERK, Akt/PKB, and cAMP, CREB, and induced BDNF, PGC-1 $\alpha$, and seladin-1 (selective Alzheimer's disease indicator-1). These macromolecules can be induced neuroprotective against oxidative injury, apoptosis, and A $\beta$-related neurotoxicity (Hwang et al., 2012; St-Pierre et al., 2006).

\section{Conclusion}

This study data indicated that the properties of Hesn could be important in the restoration of brain activity. Furthermore, Hes, as an active neuroprotectant and antioxidant, increases BDNF and TrkB genes expression in the neocortex of UPI fetal rats. Treatment with Hes offers significant protection against cerebral cortex damage in the UPI rat model. Finally, Hes may provide the beneficial therapeutic potential to treat and inhibit neurodegenerative disorders associated with oxidative stressinduced intrauterine growth restriction.

\section{Ethical Considerations}

\section{Compliance with ethical guidelines}

All applicable international, national, and institutional guidelines for the care and use of animals were followed.

\section{Funding}

This research did not receive any grant from funding agencies in the public, commercial, or non-profit sectors.

\section{Authors' contributions}

Conceptualization and supervision: Mohammad Amin Edalatmanesh; Methodology: Hamed Abdollahi and Ebrahim Hosseini; Investigation, writing - original draft, and writing - review \& editing: All authors; Data collection: Mohammad Amin Edalatmanesh and Hamed Abdollahi; Data analysis: Mohammad Amin Edalatmanesh, Hamed Abdollahi and Mohsen Foroozanfar; Funding acquisition and resources: Mohammad Amin Edalatmanesh and Hamed Abdollahi.

\section{Conflict of interest}

The authors declared no conflicts of interest.

\section{Acknowledgments}

The authors declared no acknowledgments.

\section{References}

Boschen, K. E., Criss, K. J., Palamarchouk, V., Roth, T. L., \& Klintsova, A. Y. (2015). Effects of developmental alcohol exposure vs. intubation stress on BDNF and TrkB expression 
in the hippocampus and frontal cortex of neonatal rats. International Journal of Developmental Neuroscience, 43(1), 16-24. [DOI:10.1016/j.ijdevneu.2015.03.008] [PMID] [PMCID]

Cho, J. (2006). Antioxidant and neuroprotective effects of hesperidin and its aglycone hesperetin. Archives of Pharmacal Research, 29(8), 699. [DOI:10.1007/BF02968255] [PMID]

Dekeyster, E., Geeraerts, E., Buyens, T., Van den Haute, Ch., Baekelandt, V., \& De Groef, L., et al. (2015). Tackling glaucoma from within the brain: An unfortunate interplay of BDNF and TrkB. PLoS One, 10(11), e0142067. [DOI:10.1371/journal. pone.0142067] [PMID] [PMCID]

Deodati, A., Argemí, J., Germani, D., Puglianiello, A., Alisi, A., \& De Stefanis, C., et al. (2018). The exposure to uteroplacental insufficiency is associated with activation of unfolded protein response in postnatal life. PLoS One, 13(6), e0198490. [DOI:10.1371/journal.pone.0198490] [PMID] [PMCID]

Estruel-Amades, Sh., Massot-Cladera, M., Pérez-Cano, F. J., Franch, À., Castell, M., \& Camps-Bossacoma, M. (2019). Hesperidin effects on gut microbiota and gut-associated lymphoid tissue in healthy rats. Nutrients, 11(2), 324. [DOI:10.3390/ nu11020324] [PMID] [PMCID]

Fung, C., Ke, X., Brown, A. S., Yu, X., McKnight, R. A., \& Lane, R. H. (2012). Uteroplacental insufficiency alters rat hippocampal cellular phenotype in conjunction with ErbB receptor expression. Pediatric Research, 72(1), 2-9. [DOI:10.1038/pr.2012.32] [PMID] [PMCID]

Furlan, V., Konc, J., \& Bren, U. (2018). Inverse molecular docking as a novel approach to study anticarcinogenic and anti-neuroinflammatory effects of curcumin. Molecules, 23(12), 3351. [DOI:10.3390/molecules23123351] [PMID] [PMCID]

Geva, R., Eshel, R., Leitner, Y., Fattal-Valevski, A., \& Harel, Sh. (2006). Memory functions of children born with asymmetric intrauterine growth restriction. Brain Research, 1117(1), 186-94. [DOI:10.1016/j.brainres.2006.08.004] [PMID]

Ghassabian, A., Sundaram, R., Chahal, N., McLain, A. C., Bell, E., \& Lawrence, D. A., et al. (2017). Determinants of neonatal brain-derived neurotrophic factor and association with child development. Development and Psychopatholooy, 29(4), 1499511. [DOI:10.1017/S0954579417000414] [PMID] [PMCID]

Gumus, H. G., Illa, M., Pla, L., Zamora, M., Crispi, F., \& Gratacos, E. (2018). Nutritional intra-amniotic therapy increases survival in a rabbit model of fetal growth restriction. PloS One, 13(2), e0193240. [DOI:10.1371/journal.pone.0193240] [PMID] [PMCID]

Hajialyani, M., Farzaei, M. H., Echeverría, J., Nabavi, S. M., Uriarte, E., \& Sobarzo-Sánchez, E. (2019). Hesperidin as a neuroprotective agent: A review of animal and clinical evidence. Molecules, 24(3), 648. [DOI:10.3390/molecules24030648] [PMID] [PMCID]

Hwang, S. L., Shih, P. H., \& Yen, G. C. (2012). Neuroprotective effects of citrus flavonoids. Journal of Agricultural and Food Chemistry, 60(4), 877-85. [DOI:10.1021/jf204452y]

Illa, M., Eixarch, E., Batallé, D., Arbat-Plana, A., Muñoz-Moreno, E., \& Figueras, F., et al. (2013). Long-term functional outcomes and correlation with regional brain connectivity by MRI diffusion tractography metrics in a near-term rabbit model of intrauterine growth restriction. PLoS One, 8(10), e76453. [DOI:10.1371/journal.pone.0076453] [PMID] [PMCID]
Janke, K. L., Cominski, T. P., Kuzhikandathil, E. V., Servatius, R. J., \& Pang, K. C. H. (2015). Investigating the role of hippocampal BDNF in anxiety vulnerability using classical eyeblink conditioning. Frontiers in Psychiatry, 6, 106. [DOI:10.3389/fpsyt.2015.00106] [PMID] [PMCID]

Kawser Hossain, M., Abdal Dayem, A., Han, J., Yin, Y., Kim, K., \& Kumar Saha, S., et al. (2016). Molecular mechanisms of the anti-obesity and anti-diabetic properties of flavonoids. International Journal of Molecular Sciences, 17(4), 569. [DOI:10.3390/ ijms17040569] [PMID] [PMCID]

Lim, W., \& Song, G. (2016). Stimulatory effects of coumestrol on embryonic and fetal development through AKT and ERK1/2 MAPK signal transduction. Journal of Cellular Physiology, 231(12), 2733-40. [DOI:10.1002/jcp.25381] [PMID]

Lunec, J. (1990). Free radicals: Their involvement in disease processes. Annals of Clinical Biochemistry: International Journal of Laboratory Medicine, 27(3), 173-82. [DOI:10.1177/00045632900 2700301] [PMID]

Meng, L., Liu, B., Ji, R., Jiang, X., Yan, X., \& Xin, Y. (2019). Targeting the BDNF/TrkB pathway for the treatment of tumors. Oncology Letters, 17(2), 2031-9. [DOI:10.3892/ol.2018.9854]

Miranda, M., Kent, B. A., Morici, J. F., Gallo, F., Saksida, L. M., \& Bussey, T. J., et al. (2018). NMDA receptors and BDNF are necessary for discrimination of overlapping spatial and nonspatial memories in perirhinal cortex and hippocampus. Neurobiology of Learning and Memory, 155, 337-43. [DOI:10.1016/j. nlm.2018.08.019] [PMID]

Molendijk, M. L., Spinhoven, P., Polak, M., Bus, B. A. A., Penninx, B. W. J. H., \& Elzinga, B. M. (2014). Serum BDNF concentrations as peripheral manifestations of depression: Evidence from a systematic review and meta-analyses on 179 associations (N=9484). Molecular Psychiatry, 19(7), 791-800. [DOI:10.1038/mp.2013.105] [PMID]

Parhiz, H., Roohbakhsh, A., Soltani, F., Rezaee, R., \& Iranshahi, M. (2015). Antioxidant and anti-inflammatory properties of the citrus flavonoids hesperidin and hesperetin: An updated review of their molecular mechanisms and experimental models. Phytotherapy Research, 29(3), 323-31. [DOI:10.1002/ ptr.5256] [PMID]

Pinney, S. E. (2016). Chapter 14 - Metabolic disorders and developmental origins of health and disease. In C. S. Rosenfeld (Ed.), The epigenome and developmental origins of health and disease (pp 267-289). London: Academic Press. [DOI:10.1016/ B978-0-12-801383-0.00014-1]

Raza, S. S., Khan, M. M., Ahmad, A., Ashafaq, M., Khuwaja, G., \& Tabassum, R., et al. (2011). Hesperidin ameliorates functional and histological outcome and reduces neuroinflammation in experimental stroke. Brain Research, 1420, 93-105. [DOI:10.1016/j.brainres.2011.08.047] [PMID]

Reichardt, L. F. (2006). Neurotrophin-regulated signalling pathways. Philosophical Transactions of the Royal Society B: Biological Sciences, 361(1473), 1545-64. [DOI:10.1098/rstb.2006.1894] [PMID] [PMCID]

Spulber S., Rantamäki, T., Nikkilä, O., Castrén, E., Weihe, P., \& Grandjean, Ph., et al. (2010). Effects of maternal smoking and exposure to methylmercury on brain-derived neurotrophic factor concentrations in umbilical cord serum. Toxicological Sciences, 117(2), 263-9. [DOI:10.1093/toxsci/kfq216] [PMID] [PMCID] 
St-Pierre, J., Drori, S., Uldry, M., Silvaggi, J. M., Rhee, J., \& Jäger, S., et al. (2006). Suppression of reactive oxygen species and neurodegeneration by the PGC-1 transcriptional coactivators. Cell, 127(2), 397-408. [DOI:10.1016/j.cell.2006.09.024] [PMID]

Tamilselvam, K., Braidy, N., Manivasagam, T., Essa, M. M., Prasad, N. R., \& Karthikeyan, S., et al. (2013). Neuroprotective effects of hesperidin, a plant flavanone, on rotenone-induced oxidative stress and apoptosis in a cellular model for Parkinson's disease. Oxidative Medicine and Cellular Longevity, 2013, 102741. [DOI:10.1155/2013/102741] [PMID] [PMCID]

Tashima, L., Nakata, M., Anno, K., Sugino, N., \& Kato, H. (2001). Prenatal influence of ischemia-hypoxia-induced intrauterine growth retardation on brain development and behavioral activity in rats. Neonatology, 80(1), 81-7. [DOI:10.1159/000047125] [PMID]

Verma, A., Sharma, D. K., Sarma, R., Chetia, H., \& Saikia, J. (2013). Comparative insights using the molecular homology model of BDNF (Brain Derived Neurotrophic Factor) of Varanus komodoensis and the known NGF (Nerve Growth Factor) structure of Naja atra. Bioinformation, 9(15), 755-8. [DOI:10.6026/97320630009755] [PMID] [PMCID]

Wlodek, M. E., Westcott, K. T., O'Dowd, R., Serruto, A., Wassef, L., \& Moritz, K. M., et al. (2005). Uteroplacental restriction in the rat impairs fetal growth in association with alterations in placental growth factors including PTHrP. American Journal of Physiology-Regulatory, Integrative and Comparative Physiology, 288(6), R1620-7. [DOI:10.1152/ajpregu.00789.2004] [PMID] 
This Page Intentionally Left Blank 